\title{
POLICY A perspective on health inequalities in BAME communities and how to improve access to primary care
}

\author{
Author: Oluwatosin Ajayi (Sotubo) ${ }^{\mathrm{A}}$
}

This article takes a look at access to healthcare for Black, Asian and minority ethnic (BAME) communities. Past research has shown that patients from ethnic minority backgrounds have faced inequality when accessing healthcare services. This article explores some of these reasons with a focus on primary care, including (but not limited to) language, culture, population diversity and institutional attitudes. The current reality for ethnic minority patients within our healthcare system is one which is substandard. New policies and processes should be created to tackle these issues, with ongoing quality research to further explore and monitor outcomes. With primary care being the front door to healthcare services, it must be geared to meet the needs of the whole population consistently and competently.

KEYWORDS: BAME, primary care, inequality, ethnic minority, culture

DOI: $10.7861 /$ fhj.2020-0217

\section{Introduction}

COVID-19 has brought health inequalities to the forefront more than ever before. However, for many the reality is that these inequalities and disparities are not a new phenomenon. COVID-19 has affected members of the Black, Asian and minority ethnic (BAME) communities at a shockingly disproportionate level. Public Health England's report Beyond the data: Understanding the impact of COVID-19 on BAME groups highlighted the rate of infection and mortality as being much higher for those from particular BAME communities than for their non-BAME counterparts. ${ }^{1}$

Prior to the onset of the COVID-19 pandemic, there had been evidence demonstrating poorer health outcomes and experiences for ethnic minority groups compared with the overall population. ${ }^{2}$ Examples include (but are not limited to) poor access to services and higher rates of both mental health illness and metabolic illnesses such as type 2 diabetes and cardiovascular disease. ${ }^{3}$ Genetic factors have often been considered as the primary explanation for the increased prevalence of diabetes and

Author: Ageneral practitioner, Royal College of General Practitioners, London, UK cardiovascular disease; however, there is growing evidence that this simply cannot be attributed to genetic differences alone. ${ }^{4,5}$ Now, more than ever, it is important to truly dissect these issues to find out where the problems lie within our healthcare system.

In the UK, primary care services provide the initial point of contact in the healthcare system, acting as the front door for the NHS. These services should therefore notably be a point of equal access for all in the community. However, research over the years has shown inadequate patient access and poor patient satisfaction among patients from ethnic minority backgrounds. ${ }^{6}$

This article aims to take a deeper look at the problems faced by ethnic minority communities in accessing primary care services and looks at what changes can be made to overcome these barriers to ensure the provision of equal healthcare.

It is important to also note that the term 'BAME' can be an obstacle in itself, due to fact that it can encompass a wide range of ethnicities which do not neatly fit under this umbrella definition. For the purpose of this this article, however, it predominantly refers to Black and South Asian communities.

\section{Population diversity}

To better understand the disparities, it is important to examine and recognise the increasingly diverse population of the UK. The proportion of BAME individuals rose from $8 \%$ in 2001 to $14 \%$ (by approximately 7.8 million people) of the total population in England and Wales in 2011, with no indication that this is slowing down. ${ }^{7,8}$

It has commonly been stated that the causes of health inequalities are multifactorial. These include (but are not limited to) socioeconomic class, racism/discrimination and access to healthcare. ${ }^{3}$ A 2017 resource by NHS England highlighted that certain individuals, including those insecurely housed, refugees and asylum seekers, did not always experience easy access to general practice services, and subsequently did not experience the same health outcomes as the rest of the population. ${ }^{9}$

Understanding the population at a community level and the challenges faced is visibly an imperative task in primary care. It is important that we recognise the structures and immense amount of work that has already been put in place. NHS England is responsible for commissioning high-quality primary care services for the population. ${ }^{10}$ This commissioning is shared or delegated to clinical commissioning groups (CCGs) to decide what services are needed for local populations and ensuring that they are provided. The specific requirements across CCGs primarily establish how resources are allocated. Many CCGs already have patient 
reference/participation groups as a way to involve local people in commissioning and decision making. Taking this a step further, primary care networks (PCNs) were created specifically to further address patients' needs at a community level.

Patient and public participation is an essential component of commissioning, and should continue to be considered at all stages of the commissioning cycle. It is imperative that those commissioning such services are aware of the clear aims cited by NHS England in an attempt to encourage understanding of those groups who continue to experience difficulties in accessing primary care services and consider ways to help them overcome obstacles they may encounter. ${ }^{9}$ There are evident strides being taken for further improvement, but understandably a lack of resources can make executing these improvements difficult and timely. Adequate patient access and experience can only be improved if the need for resources to support the care of BAME populations within primary care is recognised.

\section{Language}

Language plays a huge role in patient access, the patient journey and continuity of care. Therefore, it is not surprising that language barriers have been reported as the dominant factor affecting access to healthcare for those from minority ethnic communities. It is here that interpreting services play a fundamental role in the patient experience of healthcare services. ${ }^{11,12}$

General Medical Council (GMC) guidance states that 'you must make sure, wherever practical, that arrangements are made to meet patients' language and communication needs'.13 However, research has found that patients with limited English proficiency often encounter difficulties in communicating with administrative staff and that there was often a reliance on the use of informal sources of translation, such as friends and family members. ${ }^{11}$

Where difficulties in language exist, there is a need for easily accessible and reliable interpreting services. Currently, a range of models are used in primary care, varying between CCGs. Many CCGs use external commercial interpreting services, whereas other CCGs commission not-for-profit services. ${ }^{14}$

We also need to look at the difficulties faced by doctors and the impact this can have on the care given; for example, the standard 10-minute general practitioner (GP) consultation can often overrun due to added complexity and layers required. A GP often has no additional time allocated for appointments requiring a translator and understandably this can put a strain on doctors, especially for those with a higher proportion of non-Englishspeaking patients.

To care fully for patients, there needs to be adequate guidance in place and a standard set to always encourage the use of formally trained interpreters and to avoid the use of informal interpreters. The variation between CCGs, in addition to the differences in demographics and costs, suggest that further work and research are needed to establish how best to ensure equitable interpretation services within primary care. A suggestion is for practices or PCNs to manage their own budgets, and look at employing staff who can occupy multiple roles such as interpreting, advocacy and administrative roles. ${ }^{11}$ This is an interesting approach which recognises the importance of giving GPs autonomy and utilises their already established knowledge and experiences of their patient demographic. This approach also recognises the possibility of an interpreter to be multifaceted and actually part of the GP workforce, with the potential to form more of an understanding and continuity of care that could prove to be mutually beneficial.

There is also currently little evidence to show that user experience data are routinely collected. This should be part of mandatory monitoring. Due to the variety of demographics across the UK, it is logical for local CCGs to track and review their interpreter use and patient outcomes. An example of this was executed in the Southwark, Lambeth and Lewisham CCGs, which reviewed interpreting and translation services for patients in GP surgeries between October 2018 and January 2019.15 This helped to identify areas for improvement. A mandatory monitoring structure such as this should be in place to ensure that such services meet the needs of the local population. Furthermore, an understanding of the added complexity of undertaking consultations requiring formal translation is paramount, as is ensuring that additional time for such interactions becomes standard practice in order to avoid physician burnout and to maintain standards of care.

\section{Culture}

Despite language often being cited as the main barrier to accessing healthcare, it is becoming increasingly clear that cultural understanding and competence also play a part. ${ }^{16}$ So what do we mean by cultural competence? At first glance, cultural competence is about respecting and appreciating an individual's culture. It's about recognising and gaining a knowledge of different cultural practices or views and being able to understand and communicate with people across cultures. Different cultures often have entrenched beliefs and their learnt thoughts and interpretation of their health can often be very different from those of western ideals and culture. A 2014 systematic Cochrane review assessed the effects of cultural competence education interventions for healthcare professionals on patient-related outcomes; the authors concluded that there was some (limited) evidence for improved health outcomes with greater cultural competence education. ${ }^{17}$

We have to consider how a patient understands their diagnosis, their beliefs about health in general, their views and knowledge of the healthcare system and how their religious beliefs might play a role in their interactions with and access to healthcare. Systems should be put in place for healthcare providers to recognise these potential barriers and provide robust policies to tackle them. Cultural competence education training and programmes have been a topic of discussion and have, to an extent, proven beneficial; however, current research appears to be of low quality and insufficient to draw reliable conclusions. This is largely due to the size of existing studies and variety in methodology. It is clear that further research is required to establish a better understanding of cultural education interventions. ${ }^{17,18}$ However, this lack of quality research should not deter us from taking active strides to improve individual cultural understanding within the primary care workforce; I believe that striving towards greater cultural competence and understanding would be a step in the right direction.

It is once again logical for CCGs and PCNs to review the populations they serve and consider specific cultural needs in order to better understand their communities. An example would be organising patient groups in a setting that is neutral or more familiar to the local community, in order to try to understand their experiences at first hand. Furthermore, devising accessible health programmes within the community specified for ethnic minority groups could address health issues in a culturally tailored manner, 
with the aim to increase patient knowledge and understanding of the healthcare system. ${ }^{19}$

\section{Healthcare professionals' perceptions and behaviours}

One cannot explore inequality in the NHS without discussing the structural racism that exists. Structural racism is defined as "the structures, policies, practices, and norms resulting in differential access to the goods, services, and opportunities of society by race'. ${ }^{20}$

Disparities and inequality in the health service have been known about for many years. The NHS Workforce Race Equality Standard (WRES), which was established in 2015 for organisations to monitor staff experience, is a prime example. Despite these policies, BAME staff still struggle with the consequences of inequality and racism. The notorious 2015 Beyond the snowy white peaks of the NHS? report revealed the shocking lack of diversity and BAME representation at a NHS trust board/executive level. ${ }^{21}$ The 2019 WRES report found that there had been some (albeit slow) progress, with only $8.4 \%$ of board members on NHS trusts from a BAME background; a marginal increase from $7.4 \%$ in 2018 and $7.0 \%$ in $2017 .{ }^{22}$ To put this into context, as of 2019 the proportion of BAME staff working in NHS trusts was $19.7 \%$.

To improve access for ethnic minority patients, eliminating institutional racism should be a priority. Senior positions in the NHS at both national and local level should reflect the population and the society it serves. Achieving this in practice is not necessarily straightforward, particularly as there is such a diverse range of ethnicities that are not adequately represented by the term 'BAME'.

The WRES has been found to be a very useful and important tool in measuring disparities and areas of improvement within the NHS. However, it's important to highlight that, to date, the WRES is yet to take place within primary care, although policy changes for implementation are underway. It is important for the concept of the WRES to be initiated into primary care in order to examine the potential impact of workforce disparities in primary care and the impact on society.

The WRES leadership strategy in their Model employer report created a target for the NHS to reach equality in BAME representation across the workforce by 2028 , in line with the NHS long-term plan. ${ }^{23}$ So how do we go about doing this? It is assumed that individual CCGs will need to understand their workforce and patient demographics. Therefore, once the primary care WRES is in place, individual CCGs begin to develop their own plans and targets in line with guidance from the national WRES implementation team.

While there is clearly a lot of work still to be done, it should be highlighted that we are beginning to see some gradual improvements within the NHS since the WRES was implemented.

\section{Accessing primary care}

The way that patients now access primary care has fundamentally changed as a result of the COVID-19 pandemic. We have witnessed the near-overnight restructuring of the initial method of patient contact, moving from the majority of appointments being face to face to the majority now being remote consultations. While this may bring added convenience and efficiency on some levels, it also possesses the risk of enhancing some of the difficulties highlighted in this article. Language and cultural barriers can seemingly be more difficult to manage via remote consultations.
Furthermore, we must be mindful that there are those in society who do not have regular access to a computer or handheld device, or indeed are not IT literate enough to use some of the systems that have been rapidly implemented. It is certainly too early to see the effects of these changes on ethnic minorities, but it is an area that should be closely monitored to ensure safe and equitable access.

\section{Conclusion}

The diverse population of the UK is represented by various ethnic groups, each with their own distinct cultural identities and health beliefs. We have seen how poor understanding of population diversity, lack of language and cultural competence, together with institutional racism, can create barriers for patient access to primary care services.

Inequalities and disparities in healthcare are not a new problem and strategies have been attempted to tackle such issues in the past, but the lack of progress has been limited and the current COVID-19 pandemic has further highlighted the ongoing disparity in outcomes for BAME communities. The current reality facing many ethnic minority patients is one of substandard healthcare. Primary care, being the front door to healthcare services, must consistently and competently strive to meet the needs of the whole population. There remains a great deal of work to ensure that all patients, regardless of their background, receive equitable access to healthcare.

\section{References}

1 Fenton K, Pawson E, de Souza-Thomas L. Beyond the data: Understanding the impact of COVID-19 on BAME groups. Public Health England, 2020. https://assets.publishing.service.gov.uk /government/uploads/system/uploads/attachment_data/file/ 892376/COVID_stakeholder_engagement_synthesis_beyond_ the_data.pdf [Accessed 24 February 2021].

2 Harding S, Balarajan R. Limiting long-term illness among Black Caribbeans, Black Africans, Indians, Pakistanis, Bangladeshis and Chinese born in the UK. Ethn Health 2000;5:41-6.

3 Randhawa G. Tackling health inequalities for minority ethnic groups: challenges and opportunities. Better Health Briefing Paper 6. Race Equality Foundation, 2007. https://raceequalityfoundation. org.uk/housing/tackling-health-inequalities-for-minority-ethnicgroups-challenges-and-opportunities [Accessed 24 February 2021].

4 Spanakis EK, Hill Golden S. Race/ethnic difference in diabetes and diabetic complications. Curr Diab Rep 2013;13:814-23.

5 Chaturvedi N. Ethnic differences in cardiovascular disease. Heart 2003;89:681-6.

6 Smith NR, Kelly YJ, Nazroo JY. Intergenerational continuities of ethnic inequalities in general health in England. J Epidemiol Community Health 2009;63:253-8.

7 Office for National Statistics. Focus on ethnicity and identity. London: ONS, 2005.

8 Office for National Statistics. 2011 Census: key statistics for local authorities in England and Wales, March 2011. Statistical bulletin. London: ONS, 2012. www.ons.gov.uk/peoplepopulationand community/populationandmigration/ populationestimates/ bulletins/2011censuskeystatisticsforenglandandwales/2012-12-11 [Accessed 24 February 2021].

9 NHS England. Improving access for all: Reducing inequalities in access to general practice services. Leeds: NHS England, 2017. www.england.nhs.uk/publication/improving-access-for-all-reducinginequalities-in-access-to-general-practice-services [Accessed 24 February 2021]. 
10 NHS England. NHS Commissioning: primary care. NHS. www. england.nhs.uk/commissioning/primary-care [Accessed 24 February 2021].

11 Lehane D, Campion P. Interpreters: why should the NHS provide them? Br J Gen Pract 2018;68:564-5.

12 Gill PS, Shankar A, Quirke T, Freemantle N. Access to interpreting services in England: secondary analysis of national data. BMC Public Health 2009;9:12.

13 General Medical Council. Good medical practice: Domain 3: Communication, partnership and teamwork. GMC, 2019. www. gmc-uk.org/ethical-guidance/ethical-guidance-for-doctors/ good-medical-practice/domain-3---communication-partnership-and-teamwork [Accessed 24 February 2021].

14 Lehane D, Oliver P, Mitchell C, Burton C. Interpreter costs across clinical commissioning groups in England 2017-2018: a cross sectional survey using 'freedom of information' requests. $\mathrm{Br}$ ] Gen Pract 2020;70(suppl 1):bjgp20X711377.

15 NHS Lambeth Clinical Commissioning Group. Review of the primary care Interpreting and Translation Service across Lambeth, Southwark and Lewisham. NHS, 2019. www.lambethccg.nhs.uk/ get-involved/Documents/LAMBETH \% 20Evaluation \% 20report \% 20 for \% 20ITS \% 20engagement \% 20review_FINAL_190328v4.0.pdf [Accessed 24 February 2021].

16 Szczepura A. Access to health care for ethnic minority populations. Postgrad Med J 2005;81:141-7.

17 Horvat L, Horey D, Romios P, Kis-Rigo J. Cultural competence education for health professionals. Cochrane Database Syst Rev 2014;5:CD009405

18 Govere L, Govere EM. How effective is cultural competence training of healthcare providers on improving patient satisfaction of minority groups? A systematic review of literature. Worldviews Evid Based Nurs 2016;13:402-10.

19 Anderson De Cuevas RM, Saini P et al. A systematic review of barriers and enablers to South Asian women's attendance for asymptomatic screening of breast and cervical cancers in emigrant countries. BMJ Open 2018;8:e020892.

20 Jones CP. Confronting institutionalized racism. Phylon 2002;50:7-22.

21 Kline R. Beyond the snowy white peaks of the NHS? Better Health Briefing Paper 39. Race Equality Foundation, 2015. https:// raceequalityfoundation.org.uk/health-care/beyond-the-snowywhite-peaks-of-the-nhs/ [Accessed 24 February 2021].

22 The WRES Implementation Team. NHS workforce race equality standard: 2019 data analysis report for NHS trusts. NHS, 2020. www.england.nhs.uk/wp-content/uploads/2020/01/wres-2019. data-report.pdf [Accessed 24 February 2021].

23 The WRES Implementation Team. A Model Employer: Increasing black and minority ethnic representation at senior levels across the NHS. NHS, 2019. www.england.nhs.uk/wp-content/ uploads/2019/01/wres-leadership-strategy.pdf [Accessed 24 February 2021].

Address for correspondence: Dr Oluwatosin Sotubo, Royal College of General Practitioners, 30 Euston Square, London NW1 2FB, UK.

Email: o.sotubo@nhs.net

Twitter: @mindbodydoctor

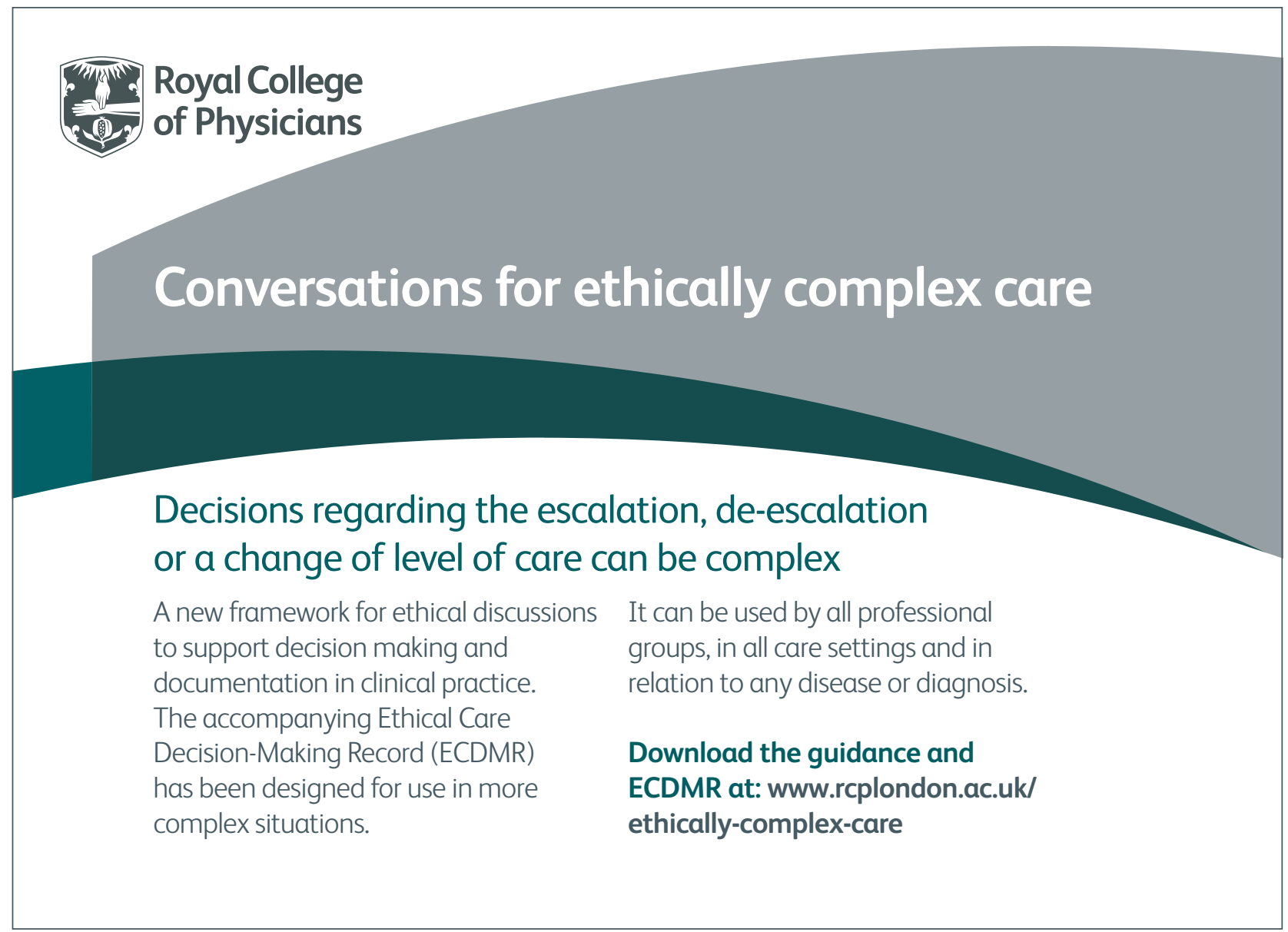

